\title{
杀结核菌素的全合成研究
}

\author{
黄海洋阮志忠胡蹈肖 强* \\ (江西科技师范大学有机功能分子研究所 南昌 330013)
}

\begin{abstract}
摘要 杀结核菌素是一个吡咯[2,3- $d$ 嘧啶核苷天然产物, 具有显著的抗血吸虫感染、抗菌和抗肿瘤活性. 报道了以微波 促进的 Vorbrüggen 糖基化反应为关键步骤，以 6-氯-7-澳-吡咯 [2,3- $d$ 嘧啶和 1-O-乙酰基-2,3,5- $O$-三苯甲酰基- $\beta$ - $D$-呋喃 核糖为原料, 经过 3 步反应以 $74 \%$ 的总收率完成了杀结核菌素的全合成. 同时研究了以全氟丁基磺酸钾、三甲基氯硅 烷和硅基化试剂[六甲基二硅胺烷或者 $N, O$-双 (三甲基硅基)乙酰胺]在微波加热下一锅法合成 7-去氮嘌呤核苷的方法. 关键词 核苷; 全合成; 天然产物; 糖基化; 微波
\end{abstract}

\section{An Improved Total Synthesis of Tubercidin}

\author{
Huang, Haiyang Ruan, Zhizhong $\quad \mathrm{Hu}$, Tao Xiao, Qiang* \\ (Jiangxi Key Laboratory of Organic Chemistry, Jiangxi Science \& Technology Normal University, Nanchang 330013)
}

\begin{abstract}
Tubercidin is a naturally occurring pyrrolo[2,3- $d]$ pyrimidine nucleoside with significantly biological activities, such as anti-schistosomal, antibacterial and antitumor. An improved total synthesis of tubercidin is reported using microwave promoted Vorbrüggen glycosylation as the key step. Thus, tubercidin was synthesized in 3 steps with $74 \%$ overal yield using 6-chloro-7-bromo-pyrrolo[2,3- $d$ ] pyrimidine and 1-O-acetyl-2,3,5-O-tribenzoyl- $\beta$ - $D$-ribose as starting materials. The application of micromave irridated one-pot reaction is also reported in the synthesis of 7-deazapurine nucleosides using potassium nonafluoro-1-butanesulfonate, trimethylsilyl chloride and silylation reagent [hexamethyldisilazane or $N, O$-bis(trimethylsilyl)acetamide].
\end{abstract}

Keywords nucleoside; total synthesis; natural product; glycosylation; microwave

杀结核菌素(1, Tubercidin, 7-deazaadenosine, 图 1) 是从杀结核链霉菌(Streptomyces tubericidus)发酵液中分 离得到的核苷抗生素 ${ }^{[1]}$, 是最早发现的 7-去氮嘌呤核苷 天然产物之一，其结构与腺苷 2(图 1)的差别仅为 N-7 改 变为 C-7. 杀结核菌素具有显著的抗血吸虫感染 ${ }^{[2]}$ 、抗 菌 ${ }^{[3]}$ 和抗肿瘤活性 ${ }^{[4]}$. 研究表明该化合物在体内通过核 苷转运机制进入细胞, 在细胞内代谢为 $5^{\prime}$-杀结核菌素 三磷酸酯, 在细胞内 DNA 和 RNA 的合成时作为腺苷三 磷酸(ATP)的类似物, 抑制 DNA 和 RNA 的合成, 从而 阻止蛋白质的表达 ${ }^{[5]} .20$ 世纪 70 年代, 杀结核菌素曾一 度作为抗癌药物用于肿瘤化疗, 在使用中发现该化合物 对癌症病人会产生中毒性肾损害的副作用, 从而终止了 其进一步临床应用 ${ }^{[6]}$.

随着分子生物学的快速发展, 杀结核菌素的生物活
性和作用机制重新得到科学家们的关注. 近期研究表 明, 杀结核菌素能够与 ATP 在微管蛋白的结合位点进 行结合来稳定微管 ${ }^{[7]}$, 而微管在维持细胞形态和辅助细 胞内运输等方面具有重要作用, 作用机理类似于抗癌药 物紫杉醇. 其衍生物, 例如 7-碘代杀结核菌素(3, 图 1) 在研究细胞内腺苷代谢时候可以作为腺苷激酶的抑制 剂，同时最近被发现是 p53 酶的激活剂 ${ }^{[8]}$.

目前杀结核菌素的主要获取途径仍然是通过从杀 结核链霸菌(Streptomyces tubericidus)的发酵液中分离得 到, 价格非常昂贵. 近年来, 我们研究小组致力于构建 天然核苷化合物库 ${ }^{[9]}$, 特别是 7-去氮核苷天然产物库, 系统研究其生源合成途径和生物活性. 以杀结核菌素为 起始原料可以合成一系列的 7-去氮核苷天然产物及其 衍生物, 因此一条高效的全合成路线就显得尤为重要.

\footnotetext{
*E-mail: xiaoqiang@tsinghua.org.cn

Received February 23, 2014; revised March 20, 2014; published online April 10, 2014.
}

Project supported by the National Natural Science Foundation of China (Nos. 20962009, 21062006), the Program for New Century Excellent Talents in University (No. 11-1000), the Training Project of Jiangxi Youth Scientists and the Bureau of Science \& Technology of Nanchang City.

国家自然科学基金(Nos. 20962009, 21062006)、教育部新世纪优秀人才支持计划(No. 11-1000)、江西省青年科学家培养对象项目和南昌市科技局资助 项目. 

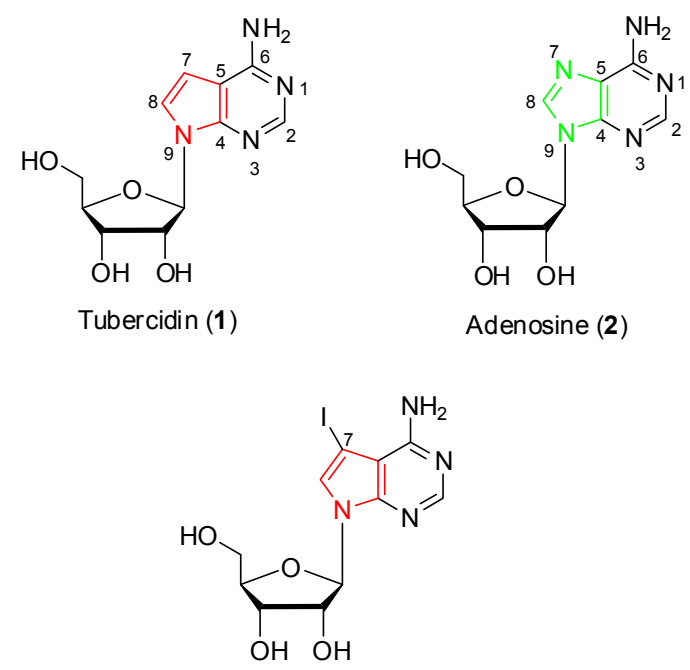

7-lodo-tubercidin (3)

图 1 杀结核菌素(1)、腺苷 2 及 7-碘代杀结核菌素(3)的结构 Figure 1 Structures of tubercidin (1), adenosine $\mathbf{2}$ and 7-iodo-tubercidin (3)

迄今为止，文献报道的杀结核菌素及其类似物的合 成几乎都是通过经典的碱基金属盐(钠盐或者钾盐)糖基 化反应进行(Scheme 1) ${ }^{[10]}$. 该方法通常需要以 $D$-核糖为 起始原料合成氯代糖 $\mathbf{4}$, 反应步骤长, 总产率低, 在 1 位 羟基氯代步骤需要用到毒性较大的六甲基磷酰三胺 (HMPA)和四氯化碳 ${ }^{[1]}$, 同时化合物 4 的稳定性较差, 使用时需要现制备, 从而影响了该方法的推广应用. 同 时早期也有通过高温熔融法合成该类化合物的报道 ${ }^{[13]}$, 但是由于反应温度高, 对底物的官能团要求苛刻, 产率 低, 实际中已经很少使用.

经过近 30 年的发展, Vorbrüggen 糖基化反应(SilylHilbert-Johnson reaction)已经成为核苷合成的标准方法 之一 ${ }^{[13]}$, 但是该反应在 7-去氮嘌呤核苷的合成中一直没 有成功, 其原因可能是由于其 N-9 位的 NH 较之正常的 嘌呤碱基(腺嘌呤或者鸟嘌呤)N-9位的 NH 碱性增强, 因
此在 Vorbrüggen 糖基化反应中不能有效进攻路易酸(通 常为三氟甲磺酸三甲基硅酯, TMSOTf)作用下形成的端 头氧嗡离子 ${ }^{[14]}$. 直到 2006 年, Seela 等 ${ }^{[15]}$ 发现在 7-去氮 嘌呤碱基的 C-7 引入吸电子的卤素原子(通常为 $\mathrm{Cl}, \mathrm{Br}$ 和 I), 可以顺利通过 Vorbrüggen 糖基化反应合成 7-去氮 腺嘌呤核苷，从而迅速推动了这类化合物的合成方法研 究. 本工作研究基于 Vorbrüggen 糖基化反应为关键步 骤合成杀结核菌素, 并对反应条件进行优化研究 (Scheme 1).

\section{1 结果与讨论}

从反合成分析(Scheme 1)可以看出, 首先需要合成 碱基 7. 该碱基可以通过 6-氯-吡咯 [2,3- $d$ ]嘧啶(9)用 NBS 进行选择性溴代，以几乎定量的产率得到，而碱基 9 以 2-氧基-4,4-二乙氧基丁酸乙酯(8)为原料可以公斤级的 制备 ${ }^{[16]}$. 接着我们研究用 Vorbrüggen 糖基化进行化合 物 11 的合成. 首先用 $N, O$-双 (三甲基硅基)乙酰胺(BSA) 对碱基 7 进行硅烷化反应, 然后分别加入糖给体 $\mathbf{1 0}$ 和 TMSOTf, 室温反应 $15 \mathrm{~min}$, 然后在 $80{ }^{\circ} \mathrm{C}$ 继续反应 $1 \mathrm{~h}$, 以 $75 \%$ 的收率顺利得到关键中间体 $\mathbf{1 1}$.

近年来，微波合成法作为一种的新的加热方式，具 有升温速度快、反应操作简单、反应速度快以及可能的 热催化效应等优点, 得到了广泛的应用 ${ }^{[18]}$, 但是该方法 在核苷糖苷化合成上的应用研究较少 ${ }^{[19]}$, 没有用于 7-去 氮嘌呤核苷的合成报道. 为了进一步研究 7-去氮嘌呤核 苷的合成，我们研究了运用微波法进行该类化合物的合 成. 同常规加热反应类似，首先用 BSA 对碱基 7 进行硅 烷化反应, 然后依次加入糖给体 $\mathbf{1 0}$ 和 TMSOTf 室温反 应 $15 \mathrm{~min}$, 然后转到微波加热条件下在 $80{ }^{\circ} \mathrm{C}$ 继续反应 $20 \mathrm{~min}$, 以 $90 \%$ 的产率得到化合物 $\mathbf{1 1}$.

一锅法反应(One-pot reaction)是一种非常有前景的 有机合成方法，可以从相对简单易得的原料出发，不经

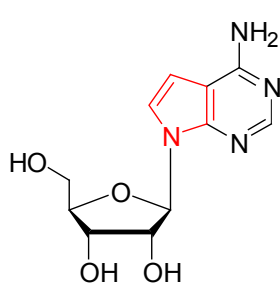

Tubercidin (1)

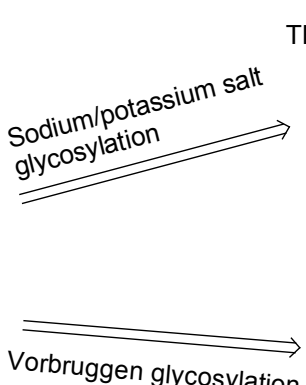

Vorbruggen glycosylation
TBDMSO

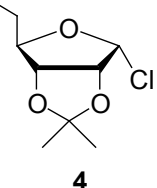

4

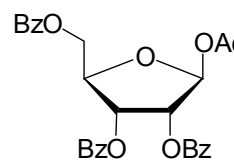

6

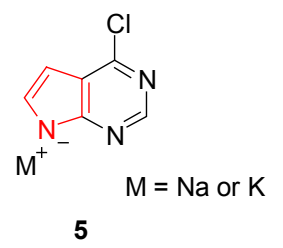<smiles>Clc1ncnc2[nH]cc(Br)c12</smiles>

7

Scheme 1 
中间体的分离，直接获得结构复杂的分子 ${ }^{[19]}$. 早在 20 世纪 80 年代, Vorbrüggen 等 ${ }^{[20]}$ 即着手研究一锅法合成核 苷化合物, 将六甲基二硅胺烷(HMDS)、全氟丁基磺酸 钾、 $\mathrm{TMSCl}$ 以及相应的碱基和糖混合加热, 以中等产率 顺利得到核苷化合物, 但是该方法并没有得到核苷化学 家的重视和应用, 可能的原因是全氟丁基磺酸钾不容易 获得的缘故. 现在, 作为聚碳酸酯材料的最佳阻然剂, 全氟丁基磺酸钾的合成工艺非常成熟，价格便宜. 我们 同时进行了一锅法合成关键中间体 11 的研究, 分别以 BSA 和 HMDS 为硅烷化试剂, 分别加入全氟丁基磺酸 钾、 $\mathrm{TMSCl}$ 、糖 10 和碱基 7, 直接微波加热 $80{ }^{\circ} \mathrm{C}$ 反应 $1 \mathrm{~h}$, 可以顺利以 $65 \%$ 和 76\%的产率得到关键中间体 11 . 同时可以以 $86 \%$ 的产率回收全氟丁基磺酸钾，再次用于 Vorbrüggen 糖基化同时不影响反应的产率. 上述反应
条件优化总结如表 1 所示，可以看出微波加热一锅法 Vorbrüggen 糖基化反应可以有效提高化合物 $\mathbf{1 1}$ 的合成 产率，同时全氟丁基磺酸三甲基硅酯作为一个可以重复 使用的路易斯酸催化剂可以成功用于 7-去氮嘌呤核苷 的合成，具有反应速度快、产率高和操作简便的优点.

接着在密封反应器中, 以新制备的氨气甲醇饱和溶 液对化合物 11 进行氨解, $130{ }^{\circ} \mathrm{C}$ 加热过夜, 在脱去苯甲 酰基保护基团的同时，取代氯原子以几乎定量的产率得 到 7-溴代杀结核菌素. 最后, 以 $20 \% \mathrm{Pd}(\mathrm{OH})_{2} / \mathrm{C}$ 为催化 剂, 加氢取代溴原子得到目标产物杀结核菌素 1 , 以化 合物 7 为原料，总产率 $74 \%$, 所有谱图数据与天然产物 一致。对杀结核菌素(1)进行亚硝化反应可以到 7-deazainosine (13)，该化合物是从海鞘分离得到的 7-去 氮核苷天然产物 ${ }^{[21]}$.<smiles>CCOC(=O)C(C)CC(OC)OC</smiles>

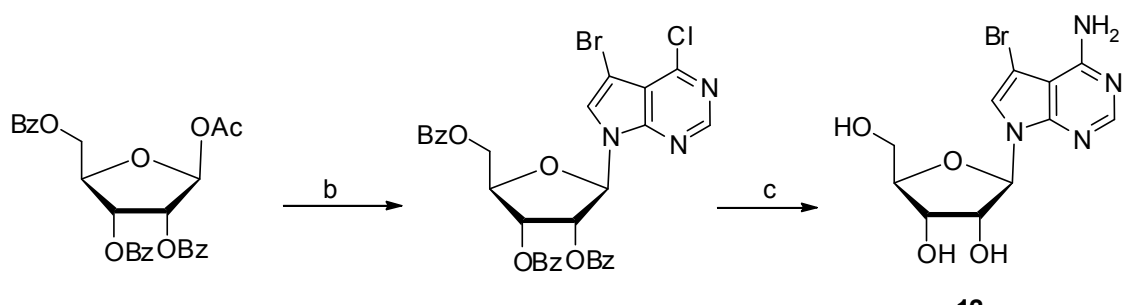

10

11

12

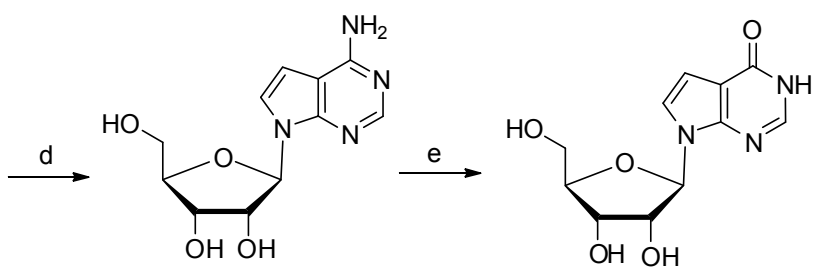

1

13

Reagents and conditions: (a) $\mathrm{CH}_{2} \mathrm{Cl}_{2}$, NBS, r.t., $12 \mathrm{~h}, 94 \%$; (b) method 1: acetonitrile, BSA, TMSOTf, $80{ }^{\circ} \mathrm{C}, 1 \mathrm{~h}, 75 \%$; method 2: acetonitrile, BSA, TMSOTF, microwave, $80{ }^{\circ} \mathrm{C}, 20 \mathrm{~min}, 90 \%$; method 3: acetonitrile, $\mathrm{BSA}, \mathrm{C}_{4} \mathrm{~F}_{9} \mathrm{SO}_{3} \mathrm{~K}, \mathrm{TMSCl}, \mathrm{microwave}, 80{ }^{\circ} \mathrm{C}, 1 \mathrm{~h}, 76 \%$; method 4 : acetonitrile, HMDS, $\mathrm{C}_{4} \mathrm{~F}_{9} \mathrm{SO}_{3} \mathrm{~K}$, TMSCl, microwave, $80{ }^{\circ} \mathrm{C}, 1 \mathrm{~h}, 65 \%$; (c) methanolic ammonia, $130{ }^{\circ} \mathrm{C}, 98 \%$; (d) $20 \% \mathrm{Pd}(\mathrm{OH}){ }_{2} / \mathrm{C}, \mathrm{THF}$, methanol, r.t., $84 \%$; (e) glacial acetic acid, $\mathrm{NaNO}_{2}, 60{ }^{\circ} \mathrm{C}, 7 \mathrm{~h}, 77 \%$

Scheme 2

表 1 Vorbrüggen 糖基化反应优化

Table 1 Optimization of Vorbrüggen glycosylation

\begin{tabular}{ccllcc}
\hline Items & Silylation reagent & \multicolumn{1}{c}{ Lewis acid } & Temperature $/{ }^{\circ} \mathrm{C}($ Time) & Heating method & Yield $/ \%$ \\
\hline Method 1 & BSA & TMSOTf & $80(1 \mathrm{~h})$ & Oil bath & 75 \\
Method 2 & BSA & TMSOTf & $80(20 \mathrm{~min})$ & Microwave & 90 \\
Method 3 & BSA & $\mathrm{C}_{4} \mathrm{~F}_{9} \mathrm{SO}_{3} \mathrm{~K}+\mathrm{TMSCl}$ & $80(1 \mathrm{~h})$ & Microwave & 76 \\
Method 4 & HMDS & $\mathrm{C}_{4} \mathrm{~F}_{9} \mathrm{SO}_{3} \mathrm{~K}+\mathrm{TMSCl}$ & $80(1 \mathrm{~h})$ & Microwave & 65 \\
\hline
\end{tabular}

${ }^{a}$ Isolated yield. 


\section{2 结论}

设计和完成了分别以 6-氯-7-溴-吡咯[2,3- $d$ 嘧啶(7) 和 1- $O$-乙酰基- $2,3,5-O$-三苯甲酰基- $\beta$ - $D$-呋喃核糖(10)为 起始原料, 以微波促进的 Vorbrüggen 糖基化反应为关 键步骤, 经过 3 步反应以 $74 \%$ 的总收率完成了杀结核菌 素的全合成, 同时首次报道了全氟丁基磺酸钾、三甲基 氯硅烷和硅基化试剂[六甲基二硅胺烷或者 $\mathrm{N}, \mathrm{O}$-双(三 甲基硅基)乙酰胺]在微波加热下一锅法合成 7-去氮嘌呤 核苷的方法, 对反应条件的进一步优化可以发展新型一 锅法合成 7-去氮腺嘌呤核苷的方法, 这些工作正在进一 步进行中.

\section{3 实验部分}

\section{1 仪器与试剂}

北京泰兴有限公司生产的 X-4 数字显示显微熔点仪 (温度计未校正); Bruker Advance DPX $400 \mathrm{MHz}$ 核磁共 振仪, 以 $\mathrm{TMS}$ 为内标, 氞代氯仿 $\left(\mathrm{CDCl}_{3}\right)$ 或者氝代二甲 亚砜( DMSO- $d_{6}$ ) 为溶剂; 电喷雾质谱(ESI-MS)为 Bruker Amazon SL 离子阱质谱仪; Anton paar autosampler MAS24 Monowave 300 微波合成仪; 常规试剂从北京东 方龙顺公司购买, 所有溶剂均为分析纯; 薄层色谱 (TLC)使用青岛化学工业公司的薄层板(GF-254), 在紫 外 $254 \mathrm{~nm}$ 波长或者用 $10 \%$ 硫酸乙醇显色进行检测; 硅 胶柱色谱使用青岛化学工业公司的硅胶(200～300 目).

\section{2 实验方法}

\subsubsection{6-氯-7-溴-吡咯 [2,3- $d$ ]嘧啶(7)的合成}

6-氯一吡咯[2,3-d]嘧啶 $(9,6.0 \mathrm{~g}, 39.1 \mathrm{mmol})$ 溶于新蒸 的无水 $\mathrm{CH}_{2} \mathrm{Cl}_{2}(200 \mathrm{~mL})$, 加入 $N$-溴代丁二酰亚胺 $(\mathrm{NBS}$, $8.1 \mathrm{~g}, 45.5 \mathrm{mmol}$ ), 室温下反应过夜, 薄层色谱(TLC)显 示反应完全, 将反应体系倒入冰水 $(500 \mathrm{~mL})$, 有大量棕 色固体析出, 将产物抽滤, 去离子水洗涤 $(200 \mathrm{~mL} \times 3)$ 后 烘干，得浅灰色固体 7 (8.5 g, 94\%). $R_{\mathrm{f}}=0.41$ $\left(\mathrm{CH}_{2} \mathrm{Cl}_{2} / \mathrm{EtOAc}, V: V=5: 1\right)$; m.p. $>300{ }^{\circ} \mathrm{C}$ [文献值 ${ }^{[17]}$ : $>300{ }^{\circ} \mathrm{C}$; ${ }^{1} \mathrm{H}$ NMR (400 MHz, DMSO- $d_{6}$ ) $\delta: 12.96$ (s, $1 \mathrm{H}), 8.60(\mathrm{~s}, 1 \mathrm{H}), 7.92(\mathrm{~s}, 1 \mathrm{H}) ;{ }^{13} \mathrm{C}$ NMR $(100 \mathrm{MHz}$, DMSO- $\left.d_{6}\right) \delta: 151.37,151.32,150.62,126.03,114.06$, 86.24; MS (ESI) $m / z: 230.1[\mathrm{M}-\mathrm{H}]^{-}$.

3.2.2 6-氯-7-溴-9-(2,3,5- $O$-三苯甲酰基- $\beta$ - $D$-呋喃核

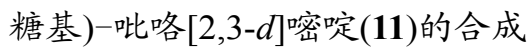

方法一: Ar气保护下, 6-氯-7-溴-吡咯[2,3- $d$ ]嘧啶(7) $(1.0 \mathrm{~g}, 4.3 \mathrm{mmol})$ 悬浮于 $40 \mathrm{~mL}$ 新蒸的无水乙腈中, 加入 BSA $(1.1 \mathrm{~g}, 5.3 \mathrm{mmol})$, 室温下反应 $10 \mathrm{~min}$, 体系变为透 明溶液, 依次加入 1- $O$-乙酰基- $2,3,5-O$-三苯甲酰基- $\beta$ - $D$ 呋喃核糖(10) (3.3 g, $65 \mathrm{mmol}$ )和 TMSOTf (1.6 g, 7.2 $\mathrm{mmol}$ ), 室温下反应 $15 \mathrm{~min}$ 后, 移入 $80{ }^{\circ} \mathrm{C}$ 的油浴中继 续反应 $1 \mathrm{~h}$, 后冷却至室温, 加入 $40 \mathrm{~mL}$ 水淬灭反应, 乙 酸乙酯萃取 $(40 \mathrm{~mL} \times 3)$, 合并有机相, 依次采用饱和碳 酸氢钠 $(40 \mathrm{~mL})$ 、饱和食盐水 $(40 \mathrm{~mL} \times 2)$ 洗涤, 无水硫酸 钠干燥 $2 \mathrm{~h}$ 后, 减压浓缩, 残余物硅胶柱层析分离纯化 得到白色固体 11 (21.8 g, 75\%).

方法二：6-氯-7-溴-吡咯[2,3- $d$ ]嘧啶(7) $(1.0 \mathrm{~g}, 4.3$ $\mathrm{mmol}$ )悬浮于 $15 \mathrm{~mL}$ 新蒸的无水乙腈中, 加入 BSA (1.1 $\mathrm{g}, 5.3 \mathrm{mmol})$, 室温下反应 $10 \mathrm{~min}$, 体系变为透明溶液, 依次加入 1- $O$-乙酰基-2,3,5- $O$-三苯甲酰基- $\beta$ - $D$-呋喃核 糖(10) (3.3 g, $6.5 \mathrm{mmol})$ 和 TMSOTf (1.6 g, $7.2 \mathrm{mmol})$, 室 温下反应 $15 \mathrm{~min}$ 后, 转入微波合成仪中在 $80{ }^{\circ} \mathrm{C}$ 条件下 反应 $20 \mathrm{~min}$, 后冷却至室温后加入 $15 \mathrm{~mL}$ 水淬灭反应, 乙酸乙酯萃取 $(15 \mathrm{~mL} \times 3)$, 合并有机相, 依次采用饱和 碳酸氢钠 $(15 \mathrm{~mL})$ 、饱和食盐水 $(15 \mathrm{~mL} \times 2)$ 洗涤, 无水 硫酸钠干燥 $2 \mathrm{~h}$ 后, 减压浓缩, 残余物硅胶柱层析分离 纯化得到白色固体 11 (2.6 g, 90\%).

方法三: $15 \mathrm{~mL}$ 新蒸的无水乙腈中, 依次加入 BSA $(1.1 \mathrm{~g}, 5.3 \mathrm{mmol}) 、 6$-氯-7-溴-吡咯[2,3- $d$ 嘧啶(7) $(1.0 \mathrm{~g}$, $4.3 \mathrm{mmol}$ )、1- $O$-乙酰基- $2,3,5-O$-三苯甲酰基- $\beta$ - $D$-呋喃核 糖(10) $(3.3 \mathrm{~g}, 6.5 \mathrm{mmol}) 、$ 全氟丁基磺酸钾 $(2.2 \mathrm{~g}, 7.2$ $\mathrm{mmol}$ )和三甲基氯硅烷 $(0.6 \mathrm{~g}, 7.2 \mathrm{mmol})$, 然后转入微波 合成仪中在 $80{ }^{\circ} \mathrm{C}$ 条件下反应 $1 \mathrm{~h}$, 后冷却至室温后加入 $15 \mathrm{~mL}$ 水淬灭反应, 乙酸乙酯萃取 $(15 \mathrm{~mL} \times 3)$, 合并有 机相, 依次采用饱和碳酸氢钠 $(15 \mathrm{~mL})$ 、饱和食盐水 $(15$ $\mathrm{mL} \times 2$ ) 洗涤, 无水硫酸钠干燥 $2 \mathrm{~h}$ 后, 减压浓缩, 残余 物硅胶柱层析分离纯化得到白色固体 11 (2.2 g, 76\%).

方法四: $15 \mathrm{~mL}$ 新蒸的无水乙腈中, 依次加入 HMDS (0.83 g, $5.3 \mathrm{mmol}), 6$-氯-7-溴-吡咯[2,3- $d$ ] 嘧啶(7) $(1.0 \mathrm{~g}, 4.3 \mathrm{mmol}), 1-O$-乙酰基-2,3,5- $O$-三苯甲酰基- $\beta$ - $D$ 呋喃核糖(10) (3.3 g, $6.5 \mathrm{mmol}) 、$ 全氟丁基磺酸钾 $(2.2 \mathrm{~g}$, $7.2 \mathrm{mmol}$ )和三甲基氯硅烷 $(0.6 \mathrm{~g}, 7.2 \mathrm{mmol})$, 然后转入 微波合成仪中在 $80{ }^{\circ} \mathrm{C}$ 条件下反应 $1 \mathrm{~h}$, 后冷却至室温后 加入 $15 \mathrm{~mL}$ 水淬灭反应, 乙酸乙酯萃取 $(15 \mathrm{~mL} \times 3)$, 合 并有机相, 依次采用饱和碳酸氢钠 $(15 \mathrm{~mL})$ 、饱和食盐水 $(15 \mathrm{~mL} \times 2)$ 洗涤, 无水硫酸钠干燥 $2 \mathrm{~h}$ 后, 减压浓缩, 残 余物硅胶柱层析分离纯化得到白色固体 11 (1.9 g, 65\%). $R_{\mathrm{f}}=0.25\left(\mathrm{CH}_{2} \mathrm{Cl}_{2} / \mathrm{PE}, V: V=5: 1\right) ;$ m.p. $142 \sim 145{ }^{\circ} \mathrm{C}$; ${ }^{1} \mathrm{H}$ NMR $\left(400 \mathrm{MHz}, \mathrm{CDCl}_{3}\right) \delta: 8.58(\mathrm{~s}, 1 \mathrm{H}), 8.11(\mathrm{~d}, J=$ $8.0 \mathrm{~Hz}, 2 \mathrm{H}), 8.00(\mathrm{~d}, J=8.0 \mathrm{~Hz}, 2 \mathrm{H}), 7.92(\mathrm{~d}, J=7.9 \mathrm{~Hz}$, $2 \mathrm{H}), 7.64 \sim 7.33(\mathrm{~m}, 10 \mathrm{H}), 6.68(\mathrm{~d}, J=5.2 \mathrm{~Hz}, 1 \mathrm{H}), 6.17 \sim$ $6.09(\mathrm{~m}, 2 \mathrm{H}), 4.90(\mathrm{~d}, J=12.1 \mathrm{~Hz}, 1 \mathrm{H}), 4.81(\mathrm{~d}, J=3.1$ $\mathrm{Hz}, 1 \mathrm{H}), 4.68(\mathrm{dd}, J=12.2,3.1 \mathrm{~Hz}, 1 \mathrm{H}) ;{ }^{13} \mathrm{C}$ NMR $(100$ $\left.\mathrm{MHz}, \mathrm{CDCl}_{3}\right) \delta: 166.14,165.40,165.10,152.73,151.63$, $150.71,133.88,133.65,129.86,129.70,129.23,128.83$, $128.62,128.57,128.32,126.49,115.93,90.18,86.71$, 
80.63, 74.09, 71.37, 63.47; MS (ESI) $m / z: 698.0[\mathrm{M}+$ $\mathrm{Na}]^{+}$.

3.2.3 6-氨基-7-溴-9-( $\beta$ - $D$-呋喃核糖基 $)$-吡咯 $[2,3-d]$ 嘧啶(12)的合成

6-氯-7-澳-9-(2,3,5- $O$-三苯甲酰基- $\beta$ - $D$-呋喃核糖 基)-吡咯[2,3- $d$ 嘧啶(11) (18.1 g, $26.9 \mathrm{mmol}$ )溶于氨气饱 和的甲醇溶液 $(400 \mathrm{~mL}), 130{ }^{\circ} \mathrm{C}$ 条件下密闭反应 $12 \mathrm{~h}$ 后, 冷却至室温, 减压浓缩, 残余物硅胶柱层析分离纯化得 白色固体 12 (9.0 g, 98\%). $R_{\mathrm{f}}=0.21\left(\mathrm{CH}_{2} \mathrm{Cl}_{2} / \mathrm{CH}_{3} \mathrm{OH}, V\right.$ : $V=20: 1$ ); m.p. $231 \sim 232{ }^{\circ} \mathrm{C}$ (文献值 ${ }^{[15 b]}: 232{ }^{\circ} \mathrm{C}$ ); ${ }^{1} \mathrm{H}$ NMR (400 MHz, DMSO- $\left.d_{6}\right) \delta: 8.10$ (s, 1H, H-2), 7.66 (s, $1 \mathrm{H}, \mathrm{H}-8), 6.81$ (br s, 2H, 6- $\mathrm{NH}_{2}$ ), 6.04 (d, $J=6.2 \mathrm{~Hz}, 1 \mathrm{H}$, H-1'), 5.35 (d, $\left.J=6.4 \mathrm{~Hz}, 1 \mathrm{H}, 2^{\prime}-\mathrm{OH}\right), 5.19 \sim 5.14(\mathrm{~m}, 2 \mathrm{H}$, $\left.3^{\prime}, 5^{\prime}-\mathrm{OH}\right), 4.36 \sim 4.32\left(\mathrm{~m}, 1 \mathrm{H}, \mathrm{H}-2^{\prime}\right), 4.09 \sim 4.06(\mathrm{~m}, 1 \mathrm{H}$, H-3'), 3.88 (d, $\left.J=3.3 \mathrm{~Hz}, 1 \mathrm{H}, \mathrm{H}-4^{\prime}\right), 3.90 \sim 3.57$ (m, 2H, $\left.\mathrm{H}-5^{\prime}\right) ;{ }^{13} \mathrm{C}$ NMR (100 MHz, DMSO- $\left.d_{6}\right) \delta: 157.42$ (C-6), 152.91 (C-2), 150.09 (C-4), 122.26 (C-8), 101.51 (C-5), 87.23 (C-1'), 87.18 (C-7), 85.64 (C-4'), 74.37 (C-2'), 70.95 (C-3'), 61.98 (C-5'); MS (ESI) $m / z: 345.1[\mathrm{M}+\mathrm{H}]^{+}$.

3.2.4 Tubercidin (1) 的合成

6-氨基-7-澳-9-(2,3,5-三羟基- $\beta$ - $D$-呋喃核糖基)-吡 咯[2,3-d]嘧啶(12) (446 mg, $1.29 \mathrm{mmol}$ ) 溶于 THF (15 mL) 和甲醇 $(15 \mathrm{~mL})$ 的混合溶液中, 加入 $20 \% \mathrm{Pd}(\mathrm{OH})_{2} / \mathrm{C}(300$ $\mathrm{mg}, 0.25 \mathrm{mmol})$, 将环境置换为 $\mathrm{H}_{2}$, 维持 $1 \mathrm{MPa}$ 压力室 温下反应 $5 \mathrm{~h}$, TLC 显示原料反应完全, 得单一产物点, 过滤除去 $\mathrm{Pd}(\mathrm{OH})_{2} / \mathrm{C}$, 减压浓缩, 残余物硅胶柱层析纯 化, 得浅黄色固体 $1(290 \mathrm{mg}, 84.1 \%) . R_{\mathrm{f}}=0.42\left(\mathrm{CH}_{2} \mathrm{Cl}_{2} /\right.$ $\mathrm{CH}_{3} \mathrm{OH}, V: V=2: 1$ ); m.p. $249 \sim 250{ }^{\circ} \mathrm{C}$ (文献值 ${ }^{[22]}$ : $244 \sim 247{ }^{\circ} \mathrm{C}$ ); ${ }^{1} \mathrm{H}$ NMR $\left(400 \mathrm{MHz}\right.$, DMSO- $\left.d_{6}\right) \delta: 8.03$ (s, 1H, H-2), 7.33 (s, 1H, H-8), 7.04 (s, 2H, 6- $\mathrm{NH}_{2}$ ), 6.57 (d, $J=3.2 \mathrm{~Hz}, 1 \mathrm{H}, \mathrm{H}-7), 5.96\left(\mathrm{~d}, J=6.2 \mathrm{~Hz}, 1 \mathrm{H}, \mathrm{H}-1^{\prime}\right), 5.31$ (s, 1H, 5'-OH), 5.25 (d, $\left.J=6.2 \mathrm{~Hz}, 1 \mathrm{H}, 2^{\prime}-\mathrm{OH}\right), 5.09$ (d, $\left.J=3.6 \mathrm{~Hz}, 1 \mathrm{H}, 3^{\prime}-\mathrm{OH}\right), 4.41$ (d, $\left.J=5.5 \mathrm{~Hz}, 1 \mathrm{H}, \mathrm{H}-2^{\prime}\right), 4.07$ (s, 1H, H-3'), 3.88 (s, 1H, H-4'), 3.62 3.50 (m, 2H, H-5'); ${ }^{13} \mathrm{C}$ NMR (100 MHz, DMSO- $d_{6}$ ) $\delta: 15.99$ (C-6), 151.98 (C-2), 150.35 (C-4), 122.75 (C-8), 103.55 (C-5), 99.98 (C-7), 88.03 (C-1'), 85.52 (C-4' ), 74.11 (C-2' ), 71.18 (C-3' ), 62.30 (C-5' ); MS (ESI) $m / z: 267.3[\mathrm{M}+\mathrm{H}]^{+}$.

\section{2 .56 -氧亚基-9-( $\beta-D$-呋喃核糖基 $)-$ 吡咯 $[2,3-d]$ 嘧} 啶(13)的合成

Tubercidin (1) (80 mg, $0.3 \mathrm{mmol}$ )溶于冰醋酸 $(7 \mathrm{~mL})$ 中, 室温条件下摚拌溶解, 逐滴加入 $\mathrm{NaNO}_{2}$ 水溶液 $\left(\mathrm{NaNO}_{2} / \mathrm{H}_{2} \mathrm{O}, 0.23 \mathrm{~g} / 2 \mathrm{~mL}\right)$, 移入 $60{ }^{\circ} \mathrm{C}$ 温度条件下反应 $7 \mathrm{~h}$. 减压浓缩, 加入 $\mathrm{CH}_{2} \mathrm{Cl}_{2}(20 \mathrm{~mL})$ 形成悬浮液, 减压 抽滤, 滤去不容物后再减压干燥, 残余物硅胶柱层析纯
化, 得白色固体 $13(62.0 \mathrm{mg}, 77 \%) . R_{\mathrm{f}}=0.29\left(\mathrm{CH}_{2} \mathrm{Cl}_{2} /\right.$ $\mathrm{CH}_{3} \mathrm{OH}, V: V=4: 1$ ); m.p. $241 \sim 243{ }^{\circ} \mathrm{C}$ (文献值 ${ }^{[23]}$ : $\left.240 \sim 243{ }^{\circ} \mathrm{C}\right) ;{ }^{1} \mathrm{H}$ NMR (400 MHz, DMSO- $\left.d_{6}\right) \delta: 11.94$ (br s, 1H, OH-6), 7.91 (s, 1H, H-2), 7.37 (d, $J=3.5 \mathrm{~Hz}$, $1 \mathrm{H}, \mathrm{H}-8), 6.53$ (d, $J=3.4 \mathrm{~Hz}, 1 \mathrm{H}, \mathrm{H}-7), 6.02$ (d, $J=6.0$ $\left.\mathrm{Hz}, 1 \mathrm{H}, \mathrm{H}-1^{\prime}\right), 5.33$ (s, 1H, 2'-OH), 5.14 (s, 1H, 3'-OH), 5.03 (s, 1H, 5'-OH), 4.32 (s, 1H, H-2'), 4.07 (s, 1H, H-3'), $3.88\left(\mathrm{~d}, J=3.4 \mathrm{~Hz}, 1 \mathrm{H}, \mathrm{H}-4^{\prime}\right), 3.62 \sim 3.51$ (m, $J=32.1$, $\left.11.5 \mathrm{~Hz}, 2 \mathrm{H}, \mathrm{H}-5^{\prime}\right) ;{ }^{13} \mathrm{C}$ NMR (100 MHz, DMSO- $\left.d_{6}\right) \delta$ : 158.64 (C-6), 148.21 (C-4), 144.22 (C-2), 121.55 (C-8), 108.79 (C-5), 102.90 (C-7), 87.43 (C-1'), 85.50 (C-4'), 74.71 (C-2'), 70.98 (C-3'), 62.01 (C-5'); MS (ESI) $m / z$ : $266.2[\mathrm{M}-\mathrm{H}]^{-}$.

\section{References}

[1] (a) Anzai, K.; Nakamura, G.; Suzuki, S. J. Antibiot., Ser. A 1957, 10, 201.

(b) Acs, G.; Reich, E.; Mori, M. Proc. Natl. Acad. Sci. U. S. A. 1964, 52, 493.

[2] (a) Jaffe, J. J.; Meymarian, E.; Doremus, H. M. Nature 1971, 230, 408.

(b) Jaffe, J. J.; Doremus, H. M.; Dunsford, H. A.; Kammerer, W. S.; Meymarian, E. Am. J. Trop. Med. Hyg. 1973, 22, 62.

[3] Bloch, A.; Leonard, R. J.; Nichol, C. A. Biochim. Biophys. Acta, Nucleic Acids Protein Synth. 1967, 138, 10.

[4] Miko, M.; Drobnica, L. Experientia 1975, 31, 832.

[5] Seibert, G.; Maidhof, A.; Zahn, R. K.; Muller, W. E. Gann 1978, 69,739

[6] (a) Smith, C. G.; Reineke, L. M.; Burch, M. R.; Shefner, A. M.; Muirhead, E. E. Cancer Res. 1970, 30, 69.

(b) Grage, T. B.; Rochlin, D. B.; Weiss, A. J.; Wilson, W. L. Cancer Res. 1970, 30, 79.

(c) Bisel, H. F.; Ansfield, F. J.; Mason, J. H.; Wilson, W. L. Cancer Res. 1970, 30, 76.

[7] Mooberry, S. L.; Stratman, K.; Moore, R. E. Cancer Lett. 1995, 96, 261.

[8] (a) Zhang, X.; Jia, D.; Liu, H.; Zhu, N.; Zhang, W.; Feng, J.; Yin, J.; Hao, B.; Cui, D.; Deng, Y.; Xie, D.; He, L.; Li, B. PLoS One 2013, 8, e62527.

(b) Ugarkar, B. G.; DaRe, J. M.; Kopcho, J. J.; Browne, C. E.; Schanzer, J. M.; Wiesner, J. B.; Erion, M. D. J. Med. Chem. 2000, $43,2883$.

[9] (a) Sun, J.; Dou, Y.; Ding, H.; Yang, R.; Sun, Q.; Xiao, Q. Mar. Drugs 2012, 10, 881 .

(b) Dou, Y.-H.; Ding, H.-X.; Yang, R.-C.; Li, W.; Xiao, Q. Chin. Chem. Lett. 2013, 24, 379.

(c) Li, Q. H.; Yang, R. C.; Ruan, Z. Z; Hu, T.; Ding, H. X.; Xiao, Q. Chin.J. Org. Chem. 2013, 33, 1340 (in Chinese).

(李启欢, 阳如春, 阮志忠, 胡蹈, 丁海新, 肖强, 有机化学, 2013, 33, 1340.)

(d) Ding, H. X.; Da, L. C.; Yang, R. C.; Cao, B. P.; Sun, Q.; Xiao, Q. Chin. Chem. Lett. 2012, 23, 996.

(e) Cao, B.; Ding, H.; Yang, R.; Wang, X.; Xiao, Q. Mar. Drugs 2012, 10, 1412.

(f) Song, Y.; Ding, H.; Dou, Y.; Yang, R.; Sun, Q.; Xiao, Q.; Ju, Y. Synthesis 2011, 1442. 
[10] (a) Kazimierczuk, Z.; Revankar, G. R.; Robins, R. K. Nucleic Acids Res. 1984, 12, 1179.

(b) Ramasamy, K.; Imamura, N.; Robins, R. K.; Revankar, G. R. J. Heterocycl. Chem. 1988, 25, 1893.

(c) Seela, F.; Muth, H.-P.; Bindig, U. Synthesis 1988, 670.

(d) Seela, F.; Westermann, B.; Bindig, U. J. Chem. Soc., Perkin Trans. 1 1988, 697.

(e) Rosemeyer, H.; Seela, F. Helv. Chim. Acta 1988, 71, 1573.

(f) Seela, F.; Kretschmer, U. J. Heterocycl. Chem. 1990, 27, 479.

(g) Seela, F.; Soulimane, T.; Mersmann, K.; Jürgens, T. Helv. Chim. Acta 1990, 73, 1879.

[11] Ramasamy, K.; Imamura, N.; Robins, R. K.; Revankar, G. R. Tetrahedron Lett. 1987, 28, 5107.

[12] (a) Tolman, R. L.; Robins, R. K.; Townsend, L. B. J. Am. Chem. Soc. 1968, 90, 524.

(b) Tolman, R. L.; Robins, R. K.; Townsend, L. B. J. Am. Chem. Soc. 1969, 91, 2102.

(c) Kondo, T.; Ohgi, T.; Goto, T. Agric. Biol. Chem. 1977, 41, 1501.

[13] (a) Niedballa, U.; Vorbruggen, H. J. Org. Chem. 1976, 41, 2084.

(b) Vorbruggen, H.; Krolikiewicz, K.; Bennua, B. Chem. Ber. 1981, 114, 1234.

(c) Teng, K.; Cook, P. D. J. Org. Chem. 1994, 59, 278.

(d) Zhong, M. H.; Robins, M. J. Tetrahedron Lett. 2003, 44, 9327.

(d) Spadafora, M.; Mehiri, M.; Burger, A.; Benhida, R. Tetrahedron Lett. 2008, 49, 3967.

(e) Vorbrüggen, H.; Ruh-Pohlenz, C. Handbook of Nucleoside Synthesis, John Wiley \& Sons, New York, 2001.

[14] Bio, M. M.; Xu, F.; Waters, M.; Williams, J. M.; Savary, K. A.;
Cowden, C. J.; Yang, C.; Buck, E.; Song, Z. J.; Tschaen, D. M.; Volante, R. P.; Reamer, R. A.; Grabowski, E. J. J. J. Org. Chem. 2004, 69, 6257.

[15] (a) Seela, F.; Peng, X. H. J. Org. Chem. 2006, 71, 81.

(b) Seela, F.; Ming, X. Tetrahedron 2007, 63, 9850.

(c) Peng, X. H.; Seela, F. Nucleosides, Nucleotides Nucleic Acids 2007, 26, 603.

[16] (a) Dillon, M. P.; Bois, D. J. D.; Lai, Y. J.; Hawley, R. C.; Wang, B. H. WO 2010066629, 2010 [Chem. Abstr. 2010, 153, 37183].

(b) Nichols, P. L.; Sehmi, S. S.; Ward, R. W.; Wilson, D. M. WO 2009080682, 2009 [Chem. Abstr. 2009, 151, 124018].

[17] (a) Xiao, S. Y.; Zhu, J.; Mu, X. J.; Li, Z. H. Chin. J. Org. Chem. 2013, 33, 1668 (in Chinese).

(肖尚友, 朱俊, 穆小静, 李正华, 有机化学, 2013, 33, 1668.)

(b) Takkellapati, S. R. Curr. Org. Chem. 2013, 17, 2305.

[18] Bookser, B. C.; Raffaele, N. B. J. Org. Chem. 2007, 72, 173.

[19] (a) Chen, B. H.; Li, J. T.; Lan, R. J. Chin. J. Org. Chem. 2013, 33, 1965 (in Chinese).

(陈保华, 李记太, 兰瑞家, 陈国锋, 有机化学, 2013, 33, 1965.)

(b) Baig, N. B. R.; Varma, R. S. Curr. Org. Chem. 2013, 17, 2323.

[20] (a) Vorbruggen, H.; Bennua, B. Chem. Ber. 1981, 114, 1279.

(b) Vorbrueggen, H. Helv. Chim. Acta 2011, 94, 947.

[21] Kim, J.; Pordesimo, E. O.; Toth, S. I.; Schmitz, F. J.; van Altena, I. J. Nat. Prod. 1993, 56, 1813.

[22] Anderson, J. D.; Bontems, R.; Geary J. S.; Cottam, H. B.; Larson, S. B.; Matsumoto, S. S.; Smee, D. F.; Robins, R. K. Nucleosides Nucleotides 1989, 8, 1201.

[23] Moreau, C.; Wagner, G. K.; Weber, K.; Guse, A. H.; Potter, B. V. L. J. Med. Chem. 2006, 49, 5162.

(Zhao, C.) 